\title{
Intravenous provocation with Neuromuscular Blocking Agents in the investigation of perioperative anaphylaxis - preliminary findings from the Danish Anaesthesia Allergy Centre(DAAC).
}

\author{
Melchiors BLB, Krøigaard M, Garvey LH \\ Danish Anaesthesia Allergy Centre, Allergy Clinic, Department of Dermatology and allergy, \\ Herlev and Gentofte Hospital, University of Copenhagen, Denmark
}

\section{Background and Goal of Study}

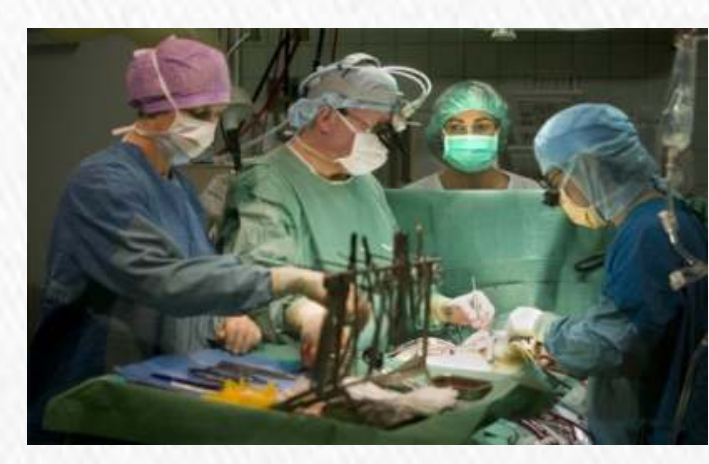

Neuromuscular blocking agents (NMBA) are considered the most common culprits in perioperative anaphylaxis (PA) in many countries. As there is crossreactivity within NMBA's, testing with several drugs is needed to identify a safe alternative. Investigation of PA have traditionally comprised in-vitro and skin testing, but due to the skin irritant properties of NMBA's the sensitivity and specificity of NMBA skintests are debated.

In drug allergy investigation provocation tests are "Gold Standard", however it is not routine in PA due to the effects of drugs used for anesthesia. In DAAC, the national reference centre for investigation of PA, drug provocation has been routinely performed for most drugs since 2004.

Since 2016 provocations have been performed with NMBA's in patients with negative or doubtful skin tests to ensure the highest accuracy of PA investigations

\section{The aim of this study is to present the preliminary findings of NMBA provocations in drug allergy investigations in DAAC.}

\section{Materials and Methods}

From 2004-2017 554 patients were investigated in DAAC.

In the period jan 2016 - july 2017 in total 49 provocations with the 4 NMBA's available in Denmark have been performed. All patients had negative or doubtful skin tests.

During provocation patients are fully monitored (ECG, saturation, BP), oxygen is administered and anesthetic back up is present in the department. Titrated provocation is done in 3 ten-fold steps up to a maximum dose of $1 / 10$ of a full dose:

cisatracurium $1 \mathrm{mg}$, suxamethonium $5 \mathrm{mg}$, rocuronium $5 \mathrm{mg}$, mivacurium $2 \mathrm{mg}$.

Maximum doses are given over 5-20 minutes by a trained anesthesiologist, and patients are closely observed for signs of allergy or effects of NMBA.

Number of NMBA provocations

since Jan 2016

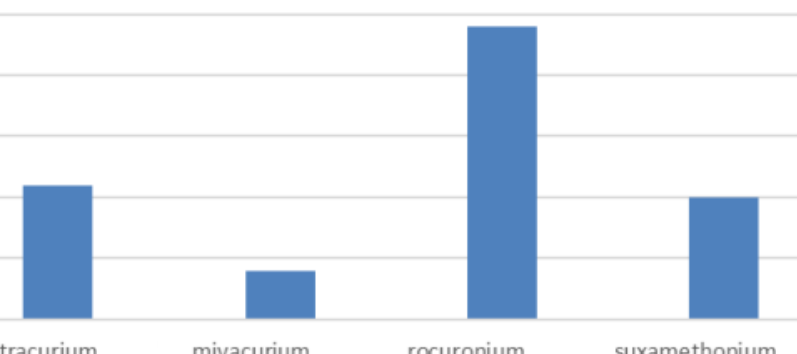

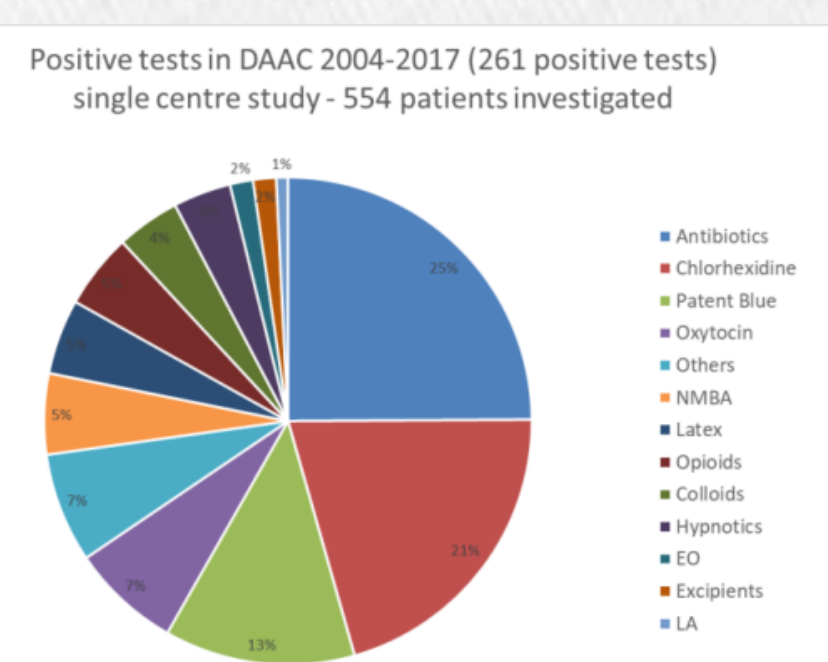

Results and Discussion

In 554 patients 261 tests were positive with the main culprits being antibiotics, chlorhexidine and patent blue.Only 14 tests (5.4\%) were positive for an NMBA on skin testing.

Out of 49 NMBA provocations only one was positive.

\section{Patients reported effects of NMBA;}

None of the patients experienced any NMBA effects at doses of $1 / 1000$ and $1 / 100$. Most patients experienced transient visual disturbance and two patients (both elderly ladies) had transient disturbance of tongue movement. None experienced respiratory difficulties or desaturation.

\section{Conclusion}

Due to the skin irritant properties of NMBA's skin testing may be difficult to interpret and may lead to false positive results restricting future choice of NMBA.

This study shows that low dose provocation with NMBA's is safe and well tolerated by patients. It should still be considered a high-risk procedure which should only be performed in highly specialized centres. 